\title{
Preparation and Charge Transport of Polyimide Insulating Nanocomposite Films
}

\author{
Junwei Zha $^{1 *}$, Zhimin Dang ${ }^{1}$ and George Chen ${ }^{2}$ \\ ${ }^{1}$ Department of Polymer Science and Engineering, School of Chemical and Biological Engineering, University of Science and Technology \\ Beijing, Beijing, 100083, China \\ ${ }^{2}$ School of Electronics and Computer Science, University of Southampton, Southampton, SO17 1BJ, United Kingdom \\ *E-mail: zjw8226@hotmail.com
}

\begin{abstract}
In order to clarify the mechanism of space charge formation and distribution in polymeric insulating materials, further experiments on preparation and charge transport of polyimide (PI) nanocomposite films were studied. Polymer nanocomposites were used in electronic and electrical engineering as a result of their excellent properties. In this paper, the $\mathrm{TiO}_{2}$ nanoparticles modified by employing surface chemical reaction were introduced into the PI matrix by using in situ polymerization. The in situ polymerization process is helpful to drive the nanoparticles into the PI matrix homogeneously. Space charge distribution in pure PI films and the $\mathrm{PI} / \mathrm{TiO}_{2}$ nanocomposite films are measured using the Pulsed Electroacoustic (PEA) method. Two process of space charge measurement were investigated, namely "volts on" and "Decay". Different types and levels of nanoparticles have been studied to understand the interaction between nanoparticles and polyimide matrix. The addition of nanoparticles has a significant influence on space charge formation, transport and decay process.
\end{abstract}

Keywords-polyimide; nanocomposite films; space charge; charge transport; PEA method

\section{INTRODUCTION}

Polymer nanocomposites have attracted much more interesting due to their enhanced properties when the inorganic nanoparticles were incorporated into polymer matrix. However, this is not a simple blending. The size, shape and content of the nanoparticles as well the state of disperse should be considered [1-2]. Polyimide (PI) is one of the best engineering plastic materials. It was widely used in domain of electrical and electronic engineering. PI nanocomposites were used as corona-resistant material which stated in the long time pulse discharge environment [3-4].

Space charge measurement is a method which can manifest the charge accumulation, distribution and transport in the dielectrics. Two kinds of charges are often used describe the nature of space charge. One is homocharge, the other one is heterocharge. The incorporations, interefaces and electrodes play important role in the space charge behavior of dielectrics. Charge injection can also be affected by surface states. The charge movement within the insulating bulk is greatly affected by the localized states. Both of them finally affect the dielectric breakdown and ageing of the dielectrics [5-6].

In this paper, pure $\mathrm{PI}$ films and $\mathrm{PI} / \mathrm{TiO}_{2}$ nanocomposite films containing surface modified nano- $\mathrm{TiO}_{2}$ particles by employing silane coupling agent were prepared using in situ dispersion polymerization process. The space charge distribution in both pure PI film and $\mathrm{PI} / \mathrm{TiO}_{2}$ nanocomposite films were investigated based on the pulsed electro-acoustic method (PEA) at room temperature. Charge dynamics in the materials during the Volts-on and decay were analyzed.

\section{EXPERIMENTAL DETAILS}

\section{A. Preparation of $\mathrm{PI} / \mathrm{TiO} \mathrm{O}_{2}$ Nanocomposite Films}

Firstly, the $\mathrm{TiO}_{2}$ nanoparticles were modified with coupling agent to improve dispersion and the interaction between the nano- $\mathrm{TiO}_{2}$ particles and polymer matrix. Secondly, in situ disperse polymerization were used to prepare $\mathrm{PI} / \mathrm{TiO}_{2}$ nanocomposite films. The in situ polymerization process is helpful to drive the nanoparticles into the polymer matrix homogeneously. The procedures of preparing the PI/inorganic nanoparticle nanocomposite films were presented in the previous literature [7] in which in-situ polymerization was adopted. Microstructure of the $\mathrm{PI} / \mathrm{TiO}_{2}$ nanocomposite films was studied by scanning electron microscopy (SEM, HITACHI S-4700) as shown in Fig. 1. SEM images reveal a homogeneous dispersion of nanoparticles in the PI matrix and all of the particles are separate in size of nanoscale.

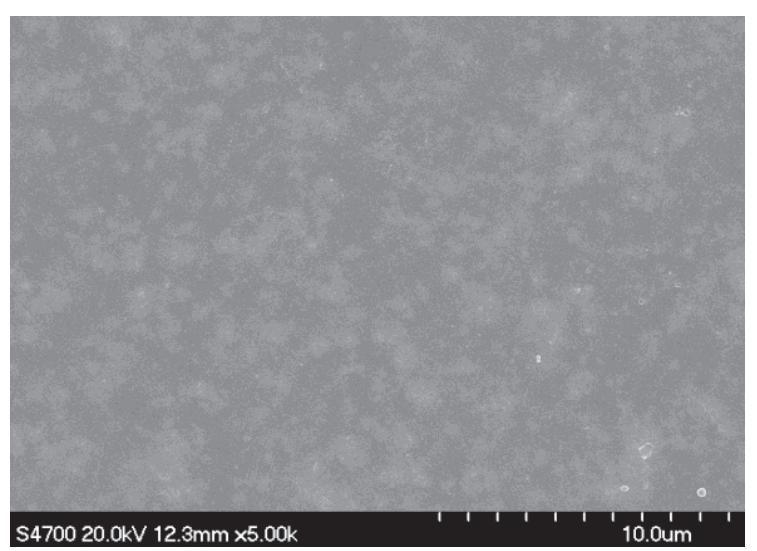

Figure 1. SEM image of PI nanocomposite films

\section{B. Space Charge Measurement}

In the past several decades, many techniques were developed to measure the space charge distribution in dielectrics. Nowadays, the Pulsed Electroacoustic (PEA) method has been widely used by researchers around the word. In this paper, the PEA method was used for measuring the dynamic space charge distribution in pure PI films and PI nanocomposite films. Fig. 2 shows the testing system of PEA in our lab. All the samples were cleaned by alcohol before tested. Each sample was placed between semiconductor (anode) and aluminum (cathode) electrodes. To improve acoustic coupling and eliminate air gap, a thin layer of silicone oil was formed between the sample and electrodes. In 
this experiment, the PEA tests were performed on a single layer sample with a thickness of $\sim 60 \mathrm{um}$ at room temperature.

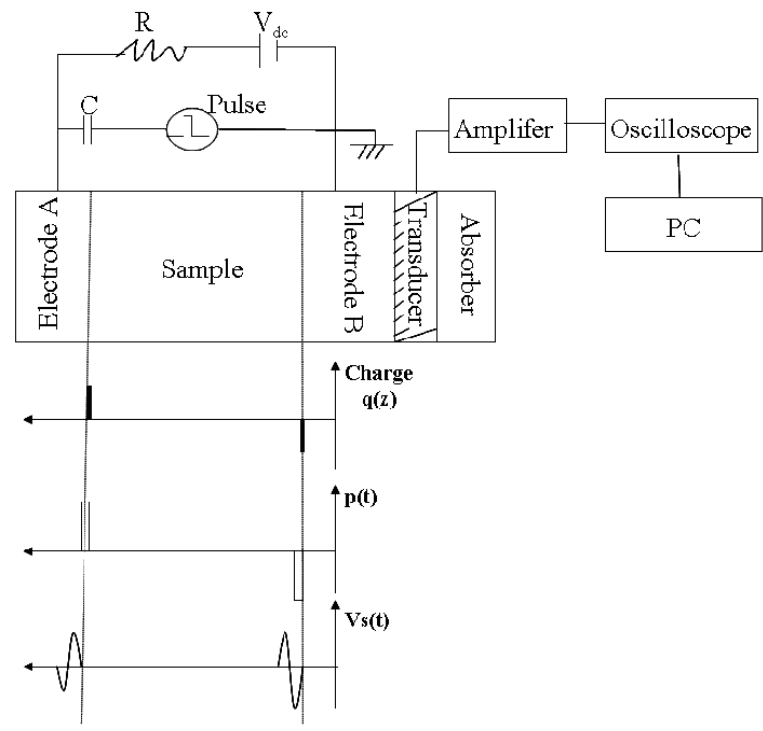

Figure 2. Schematic diagram for the PEA testing system

\section{RESULTS AND DISCUSSION}

In the whole testing process, the experiment consists of two parts: "volts-on" and "Decay" of space charge measurements. The voltage was applied to the sample for $1 \mathrm{~h}$ and space charge distributions were measured at various times. After $1 \mathrm{~h}$ dc stressing, charge decay after the removal of the applied voltage was monitored for further $1 \mathrm{~h}$. The voltage level was about $2 \mathrm{kV}$, resulting in that the applied electric field was about $33 \mathrm{kV} / \mathrm{mm}$.

\section{A. The Volts-on of space charge measurement}

Fig. 3 shows space charge distributions in the samples with different concentration of the nano- $\mathrm{TiO}_{2}$ particles loading for $30 \mathrm{~s}, 10 \mathrm{~min}, 30 \mathrm{~min}$ and $60 \mathrm{~min}$, respectively. Electrodes positions are marked by dotted lines in Fig. 3 (a), (b) and (c). The distance between the lines presents the sample thickness. At a voltage of $2 \mathrm{kV}$ charge can be found forming in all the samples. It also can be seen that the amount of charge in the sample increases with the duration of the applied voltage.

In pure PI films, bipolar charge injection has taken place. Charges from both electrodes have been observed but the amount of the injected charge from the cathode seems more in pure PI films. The charge formation in samples with nano$\mathrm{TiO}_{2}$ particles is different from the pure PI films. Heterocharges are formed adjacent to the cathode, indicating the influence of the nano- $\mathrm{TiO}_{2}$ particles on charge formation. Generally, both charge injection and ionization process which is related to the nano- $\mathrm{TiO}_{2}$ particles occurred in the samples. Actually, the injection can be seen in the region adjacent to the anode but the amount of charge is small, thus it may be masked by charges from ionization.
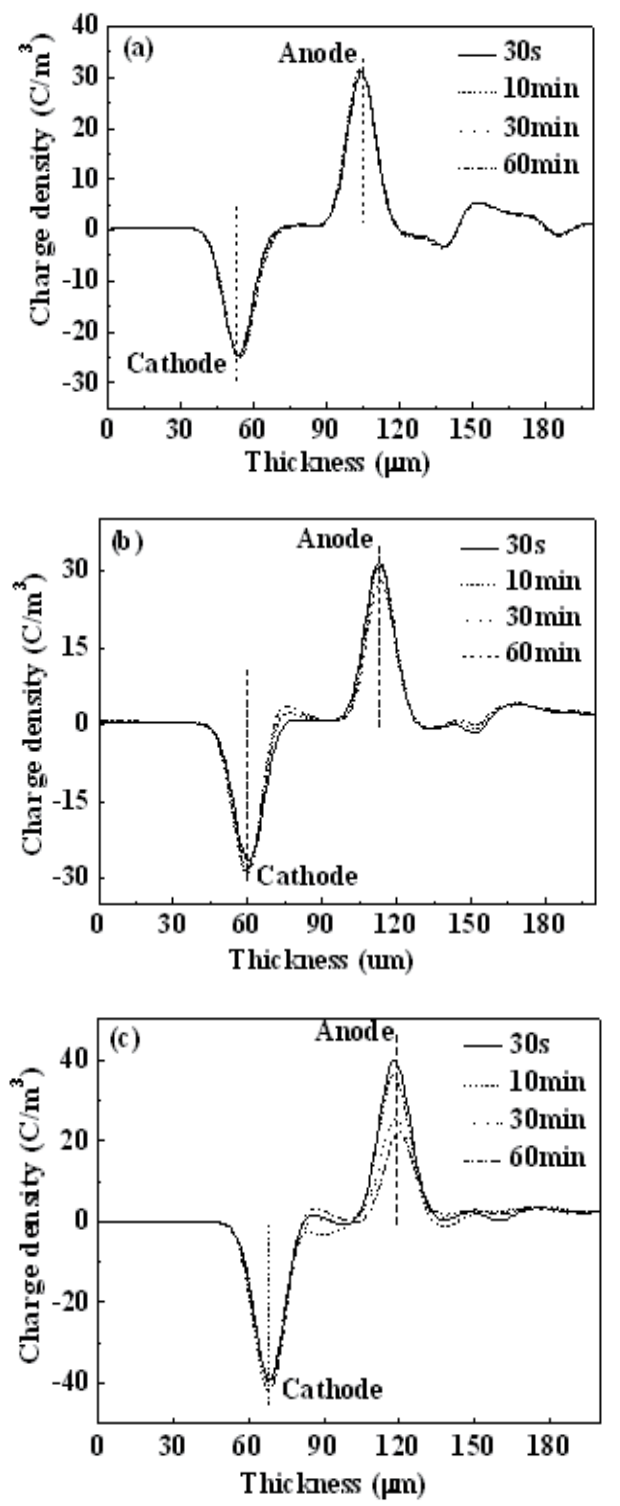

Figure 3. Space charge distribution build-up in different samples at 2kv: (a) pure PI film, (b) $\mathrm{PI} / \mathrm{TiO}_{2}-15$ wt.\% nanocomposite film, (c) $\mathrm{PI} / \mathrm{TiO}_{2}-20$ wt.\% nanocomposite film, respectively.

\section{B. The decay of space charge measurement}

The applied voltage was removed and the electrodes were short circuited after $1 \mathrm{~h}$ dc stress. Space charge distribution and trap depths in the $\mathrm{PI} / \mathrm{TiO}_{2}$ nanocomposite films as a function of time in short circuit were studied as shown in Fig. 4. It can be observed that the accumulation of space charge near the electrodes weakens with increasing the decay time as shown in Figs.4 (a), (b) and (c). The nano- $\mathrm{TiO}_{2}$ particles have taken great effect on decay of space charge in PI nanocomposite films. Fig. 4 (b) shows that the accumulation of space charge near the cathode in the $\mathrm{PI} / \mathrm{TiO}_{2}$ nanocomposite films with the concentration of nano- $\mathrm{TiO}_{2}$ particles up to $15 \mathrm{wt} \%$ is relatively low. 

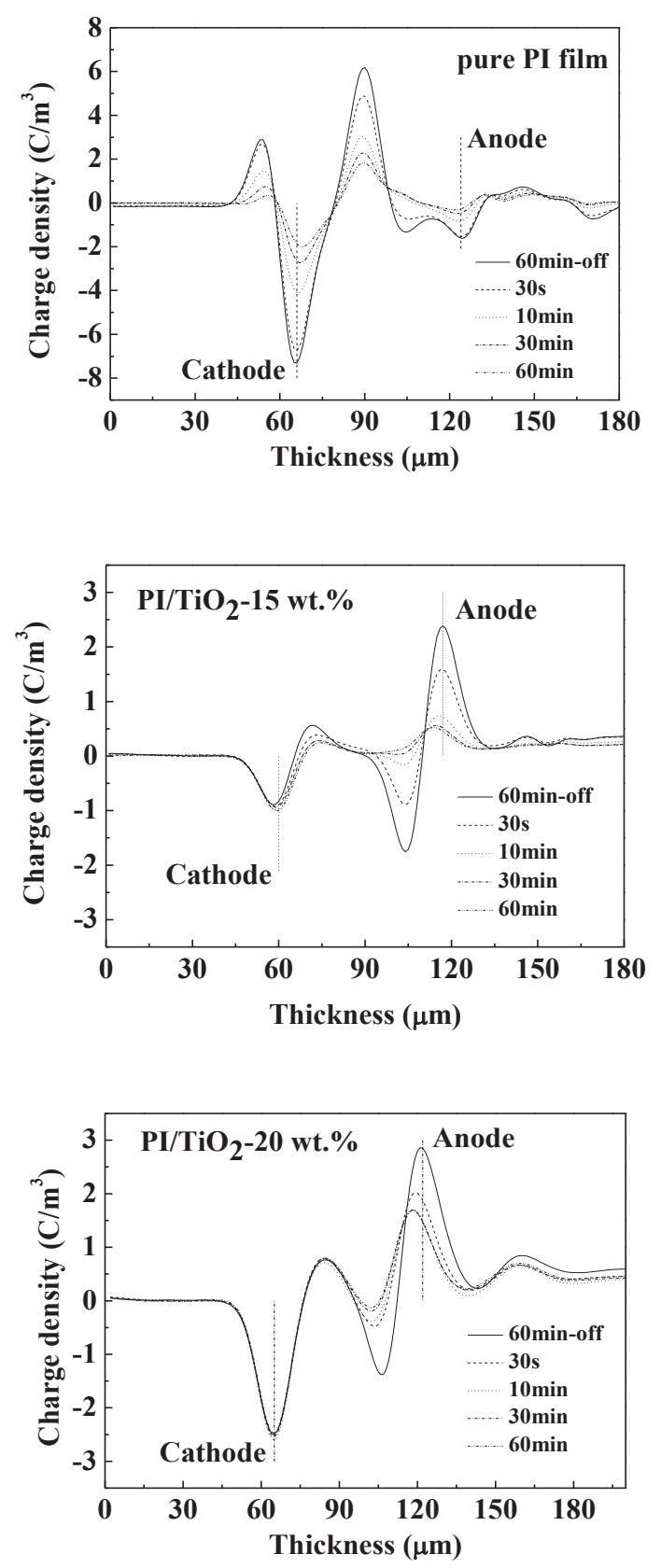

Figure 4. Space charge distributions in the films after removing the applied electrical stress

\section{CONCLUSION}

The main conclusions are as follows.

By employing in situ dispersive polymerization, the polyimide nanocomposite films loaded with different concentration of the nano- $\mathrm{TiO}_{2}$ particles were fabricated. A homogeneous dispersion of nanoparticles in the PI matrix was observed.

The space charge dynamics in these nanocomposite films have been investigated by using the PEA technique. The amount of space charge injected increases with the duration of applied voltage. The space charge characteristics in nanocomposites containing the nano- $\mathrm{TiO}_{2}$ particles are very different from the pure PI sample. Heterocharges dominate the distribution indicating ionization occurrence.

Decay of space charge in the samples anticipated that the electrical performance of $\mathrm{PI} / \mathrm{TiO}_{2}$ nanocomposite films will be different from the pure PI films. The space charge accumulation in $\mathrm{PI} / \mathrm{TiO}_{2}$ nanocomposite films decays faster than that in pure PI films due to the conduction of the nano$\mathrm{TiO}_{2}$ particles.

\section{ACKNOWLEDGMENT}

This work was financially supported by NSF of China (Grant No. 50977001 and 51073015), and State Key Laboratory of Power System (Grant No. SKLD09KZ03). This work was also supervised by Prof. George Chen.

\section{REFERENCES}

[1] T. Tanaka, G. C. Montanari, and R. Mulhaupt, "Polymer nanocomposites as dielectrics and electrical insulation-perspectives for processing technologies, material characterization and future applications," IEEE Trans. Dielectr. Electr. Insul., Vol. 11, pp. 763 784, 2004.

[2] P. Barber, S. Balasubramanian, Y. Anguchamy, S. Gong, A. Wibowo, H. Gao, H. J. Ploehn, and H. C. Loye, "Polymer composite and nanocomposite dielectric materials for pulse power energy storage," Material, Vol. 2, pp. 1697-1733, 2009.

[3] P. C. Chiang and W. T. Whang, "The synthesis and morphology characteristic study of BAO-ODPA polyimide/ $/ \mathrm{TiO}_{2}$ nano hybrid films," Polymer, Vol. 44, pp. 2249-2254, 2003.

[4] Y. H. Zhang, S. G. Lu, Y. Q. Li, Z. M. Dang, J. H. Xin, S. Y. Fu, G. T. Li, R. R. Guo, and L. F. Li, "Novel silica tube/polyimide composite films with variable low dielectric constant", Adv. Mater., Vol. 17, pp. 1056-1059, 2005.

[5] G. Chen, M. Fu, X. Z. Liu, and L. S. Zhong, "Ac aging and spacechargecharacteristic in low-density polyethylene polymeric insulation," J. Appl. Phys., Vol. 97, pp. 083713-1-4,2005.

[6] G. Chen, Y. Tanaka, T. Takada, and L. zhong, "Effect of polyethylene interface on space charge formation," IEEE Transl. Dielec. Electr. Insul., Vol. 11, pp. 113-121,2004.

[7] J. W. Zha, Z. M. Dang, H. T. Song, Y. Yin, and G. Chen. "Dielectric properties and effect of electrical aging on space charge accumulation in polyimide $/ \mathrm{TiO}_{2}$ nanocomposite films," J. Appl. Phys., Vol.108, pp. 094113(1-6), 2010. 